\title{
Statin Use in COVID-19 Hospitalized Patients and Outcomes: A Retrospective Study
}

\section{OPEN ACCESS}

Edited by:

Mualla Ozcan,

Washington University School of Medicine in St. Louis, United States

Reviewed by: Damien Garçon, Montreal Clinical Research Institute (IRCM), Canada

Prashant Nedungadi, American Heart Association, United States

*Correspondence:

Amirhossein Sahebkar sahebkara@mums.ac.ir; amir_saheb2000@yahoo.com Maciej Banach

maciej.banach@icloud.com

Specialty section

This article was submitted to Lipids in Cardiovascular Disease, a section of the journal Frontiers in Cardiovascular Medicine

Received: 22 November 2021 Accepted: 19 January 2022

Published: 24 February 2022

Citation:

Kouhpeikar H, Khosaravizade Tabasi H, Khazir Z, Naghipour A, Mohammadi Moghadam $\mathrm{H}$,

Forouzanfar $H$, Abbasifard $M$, Kirichenko TV, Reiner Ž, Banach M and Sahebkar A (2022) Statin Use in COVID-19 Hospitalized Patients and Outcomes: A Retrospective Study. Front. Cardiovasc. Med. 9:820260. doi: 10.3389/fcvm.2022.820260

\author{
Hamideh Kouhpeikar ${ }^{1}$, Hamidreza Khosaravizade Tabasi ${ }^{2}$, Zahra Khazir' ${ }^{2}$, \\ Armin Naghipour ${ }^{3}$, Hussein Mohammadi Moghadam², Hasan Forouzanfar ${ }^{2}$, \\ Mitra Abbasifard ${ }^{4,5}$, Tatiana V. Kirichenko ${ }^{6}$, Željko Reiner ${ }^{7}$, Maciej Banach ${ }^{8,9 *}$ and \\ Amirhossein Sahebkar ${ }^{10,11,12,13 *}$
}

\begin{abstract}
'Department of Hematology and Blood Bank, Tabas School of Nursing, Birjand University of Medical Science, Birjand, Iran, ${ }^{2}$ Department of Nursing, Tabas School of Nursing, Birjand University of Medical Science, Birjand, Iran, ${ }^{3}$ Department of Biostatistics and Epidemiology, Hamadan-Iran Clinical Research Development Center, Imam Reza Hospital, Hamadan University of Medical Sciences, Kermanshah University of Medical Sciences, Kermanshah, Iran, ${ }^{4}$ Immunology of Infectious Diseases Research Center, Research Institute of Basic Medical Sciences, Rafsanjan University of Medical Sciences, Rafsanjan, Iran, ${ }^{5}$ Department of Internal Medicine, Ali-Ibn Abi-Talib Hospital, School of Medicine, Rafsanjan University of Medical Sciences, Rafsanjan, Iran, ${ }^{6}$ Laboratory of Cellular and Molecular Pathology of Cardiovascular System, AP Avtsyn Research Institute of Human Morphology, Moscow, Russia, ${ }^{7}$ Department of Internal Medicine, School of Medicine, University Hospital Centre Zagreb, University of Zagreb, Zagreb, Croatia, ${ }^{8}$ Department of Preventive Cardiology and Lipidology, Chair of Nephrology and Hypertension, Medical University of Lodz, Łódź, Poland, ${ }^{9}$ Cardiovascular Research Centre, University of Zielona Gora, Zielona Gora, Poland, ${ }^{10}$ Applied Biomedical Research Center, Mashhad University of Medical Sciences, Mashhad, Iran, "11 Biotechnology Research Center, Pharmaceutical Technology Institute, Mashhad University of Medical Sciences, Mashhad, Iran, ${ }^{12}$ School of Medicine, The University of Western Australia, Perth, WA, Australia, ${ }^{13}$ Department of Biotechnology, School of Pharmacy, Mashhad University of Medical Sciences, Mashhad, Iran
\end{abstract}

Background: Coronavirus disease 2019 (COVID-19) might affect everyone, but people with comorbidities such as hypertension and cardiovascular disease (CVD) may often have more severe complications and worse outcomes. Although vaccinations are being performed worldwide, it will take a long time until the entire population of the world is vaccinated. On the other hand, we are witnessing the emergence of new variants of this virus. Therefore, effective therapeutic approaches still need to be considered. Statins are well-known lipid-lowering drugs, but they have also anti-inflammatory and immunomodulatory effects. This study aimed to investigate the effects of statins on the survival of COVID-19 hospitalized patients.

Methods: This retrospective study was performed on 583 patients admitted to a highly referenced hospital in Tabas, Iran, between February 2020 and December 2020. One hundred sixty-two patients were treated with statins and 421 patients were not. Demographic information, clinical signs, and the results of laboratory, and comorbidities were extracted from patients' medical records and mortality and survival rates were assessed in these two groups.

Results: The results of the Cox crude regression model showed that statins reduced mortality in COVID-19 patients ( $\mathrm{HR}=0.56,95 \% \mathrm{Cl}: 0.32,0.97 ; p=0.040)$, although this reduction was not significant in the adjusted model $(H R s=0.51,95 \% \mathrm{Cl}: 0.22,1.17$; $p=0.114)$. Using a composite outcome comprising intubation, ICU admission, and mortality, both crude $(\mathrm{HR}=0.43 ; 95 \% \mathrm{Cl}: 0.26,0.73 ; p=0.002)$ and adjusted (HR $=0.57 ; 95 \% \mathrm{Cl}: 0.33,0.99 ; p=0.048)$ models suggested a significant protective effect of statin therapy. 
Conclusion: Due to anti-inflammatory properties of statins, these drugs can be effective as an adjunct therapy in the treatment of COVID-19 patients.

Keywords: COVID-19, statin, mortality, ICU, inflammation

\section{INTRODUCTION}

The new SARS-CoV-2 corona virus causing COVID-19 disease is responsible for the COVID 19 pandemic. This virus causes, among other harmful effects, an inflammatory condition leading to acute respiratory distress syndrome (ARDS) in patients (1). Everyone is at risk for complications of COVID-19, but people with comorbidities, such as pulmonary diseases, malignant diseases, cardiovascular diseases, hypertension, dyslipidemia, and diabetes are considered as a high-risk group that may have more severe complications. Statins or 3-hydroxy-3methylglutaryl coenzyme A reductase (HMG-CoA) inhibitors are primarily lipid-lowering drugs. Statins do not only decrease elevated total and LDL-cholesterol in serum and therefore are effective in preventing atherosclerotic cardiovascular disease but also have pleiotropic effects. Their protective effects include anti-inflammatory, antioxidant, antithrombotic, and immune modulatory effects (2-8).

Studies have reported that statins may inhibit COVID-19 infection by binding to a key virus protease (Mpro). This protease plays an important role in proteolytic maturation (9). Coronaviruses also stimulate an inflammatory cascade by interaction with Toll-like receptors on the membrane of host cells and activating of NF- $\kappa$ B pathways. Therefore,

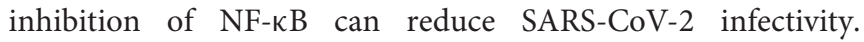
On the other hand, it has been demonstrated that statins significantly suppressed the activation of NF- $\kappa \mathrm{B}$ and exert anti-inflammatory actions (10-12). Observational studies have reported that treatment with statins was associated with a reduced mortality rate in hospitalized patients with influenza (13). Previous studies have shown that statins may be also useful in the treatment of COVID-19 due to their pleotropic effect (14-18).

In addition, since atherosclerotic cardiovascular disease and dyslipidemia are associated with poor prognosis in COVID-19 patients, statins may be also useful in improving the treatment of these patients by reducing the complications of the disease (19). Several mechanisms have been suggested for the efficacy of statins in COVID-19. CD 147, a receptor for SARS-CoV-2 on the membrane of host cells, is a novel route for SARS-CoV-2 invasion and it has been shown that statins could reduce the infectivity of SARS-CoV-2 by reducing the expression of this receptor (20, 21). Although almost all countries have started with vaccination against SARS-CoV-2, due to the emergence of mutated variants of this virus and differences in vaccination rates in different countries, it is not clear when this pandemic will stop. Therefore, looking for more effective therapies for COVID-19 patients will remain an important strategy. Acute respiratory syndrome and thromboembolism are two well-known complications that cause mortality in most of the hospitalized COVID-19 patients (22). Evidence suggests that these complications of COVID-19 are associated with an inflammatory response (23). Recent studies demonstrated that statins reduced the mortality rate by $42 \%$ in hospitalized patients with COVID-19 (24). Statins also decreased the risk of acute respiratory distress syndrome (ARDS) (25). On the other hand, some studies have reported that statins may increase the expression of ACE2 (angiotensin converting enzyme 2) and ACE2 is a receptor for SARS-CoV-2 enabling the virus to enter the host cells $(26,27)$. Therefore, inhibiting the renin-angiotensin system cascade, which is a target of ACE2, might also have some effects in patients with COVID-19 who have cardiovascular disease (28). Anyhow, according to different studies, there are still controversies about the effectiveness of statins in COVID-19 patients. Therefore, the aim of this retrospective study was to investigate the relationship between treatment with statins and the survival rate in hospitalized COVUD-19 patients.

\section{MATERIALS AND METHODS}

\section{Study Design}

A retrospective study was performed on COVID-19 patients admitted to Shahid Mostafa Khomeini Hospital in Tabas, Iran. Participants were admitted between February 2020 and December 2020. A total of 583 COVID-19 patients with symptoms of the disease and positive polymerase chain reaction (PCR) were included in the study. One hundred sixty-two patients were treated with statins and 421 were in the nonstatin group.

Patients in the statin group were all treated with atorvastatin $40 \mathrm{mg}$ daily.

Demographic characteristics, patients' clinical signs and symptoms, comorbidities, laboratory test results, chest computed tomography (CT) scan reports, vital signs, and clinical outcomes were collected from medical patients' records. Laboratory test results included complete blood count (CBC), liver enzymes, urea, creatinine, CRP, and vital signs such as body temperature, spO2, and blood oxygen level. The data on oxygen therapy, mechanical ventilation, and length of hospital stay were collected from medical records. All data were checked by two separate researchers to verify their accuracy.

The endpoints for evaluating the patients' status included the need for oxygen therapy, hospitalization in the intensive care unit (ICU), blood oxygen level, duration of hospitalization, and mortality rate. A secondary composite outcome comprising mortality, ICU admission, and intubation was also considered. Exclusion criteria were: people over 85 years and under 19 years and people with these diseases: hepatitis, AIDS, influenza, and tuberculosis.

This study was approved by the ethics committee of Birjand University of Medical Sciences (IR.BUMS.REC.1400.073). 
TABLE 1 | Characteristics of patients in statin and non-statin groups.

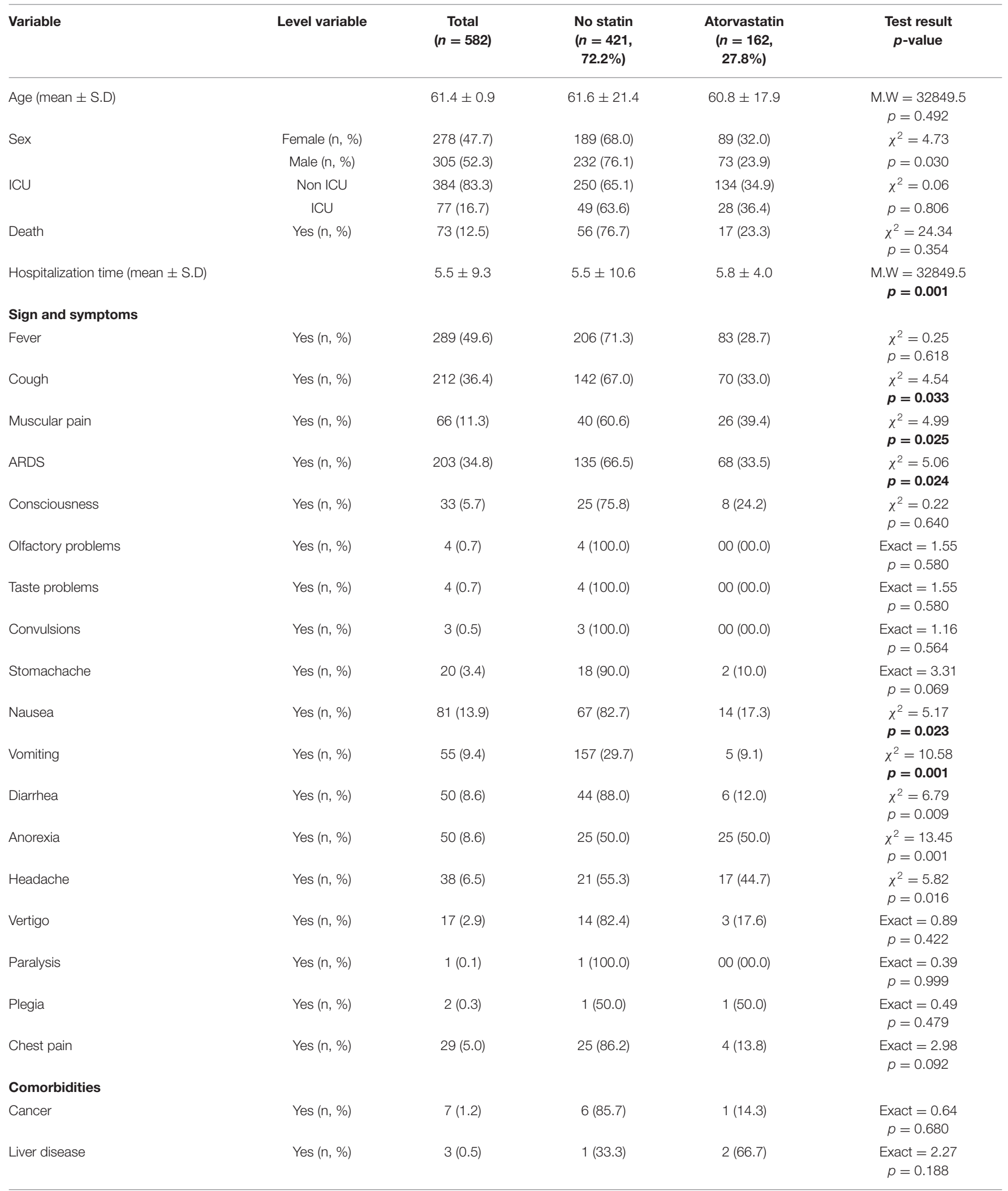


TABLE 1 | Continued

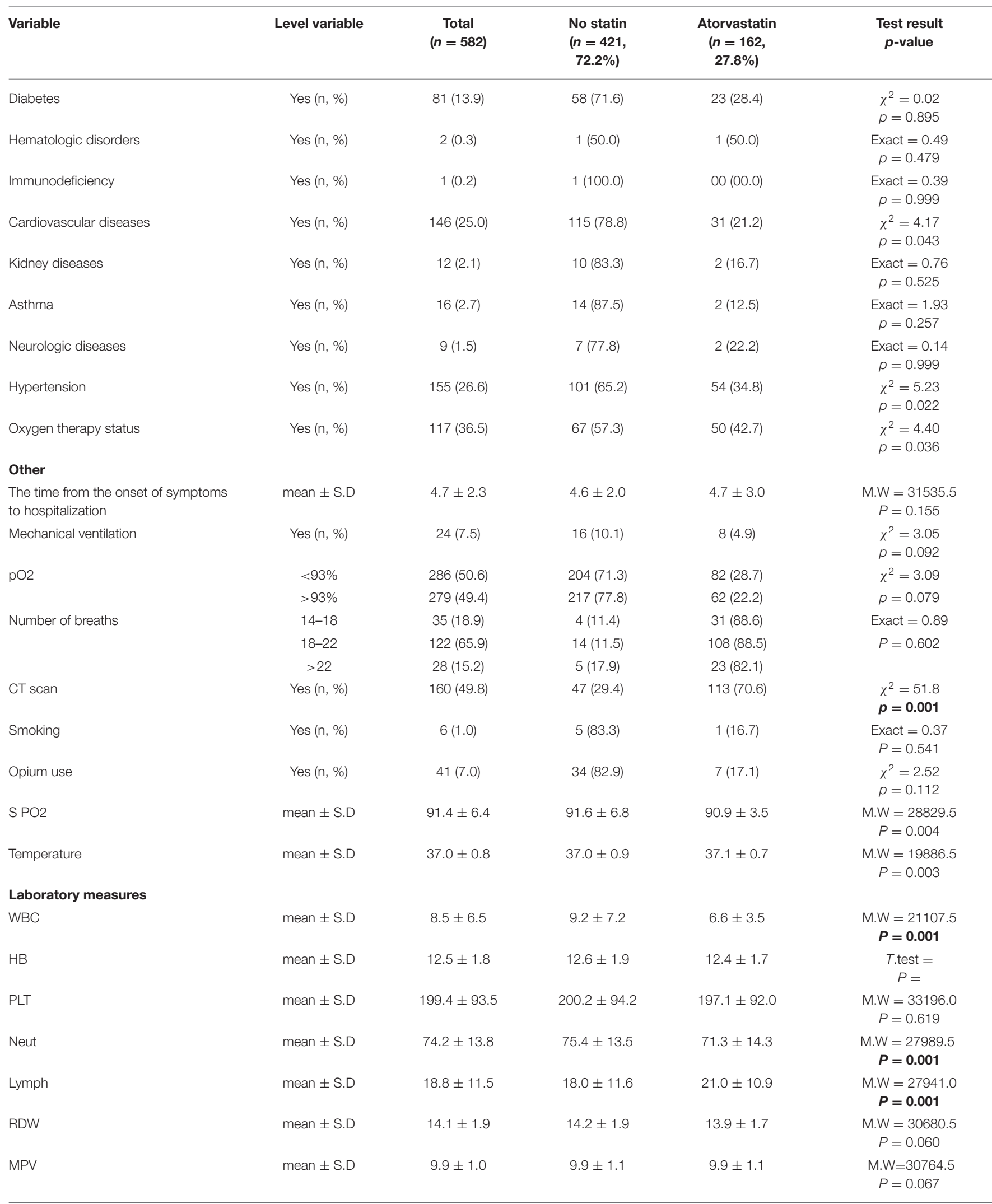


TABLE 1 | Continued

\begin{tabular}{|c|c|c|c|c|c|}
\hline Variable & Level variable & $\begin{array}{c}\text { Total } \\
(n=582)\end{array}$ & $\begin{array}{c}\text { No statin } \\
(n=421, \\
72.2 \%)\end{array}$ & $\begin{array}{l}\text { Atorvastatin } \\
\qquad(n=162 \\
27.8 \%)\end{array}$ & $\begin{array}{c}\text { Test result } \\
p \text {-value }\end{array}$ \\
\hline BUN & mean \pm S.D & $17.4 \pm 12.3$ & $17.6 \pm 13.1$ & $16.7 \pm 9.9$ & $\begin{aligned} M . W & =33235.0 \\
P & =0.634\end{aligned}$ \\
\hline $\mathrm{Cr}$ & mean \pm S.D & $1.2 \pm 0.9$ & $1.2 \pm 0.7$ & $1.3 \pm 1.4$ & $\begin{aligned} M . W & =30424.5 \\
P & =0.042\end{aligned}$ \\
\hline CRP & mean \pm S.D & $1.1 \pm 0.7$ & $1.2 \pm 0.8$ & $0.9 \pm 0.3$ & $\begin{aligned} M . W & =25494.0 \\
\boldsymbol{P} & =\mathbf{0 . 0 0 1}\end{aligned}$ \\
\hline
\end{tabular}

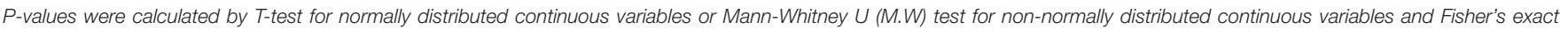
(exact) test or $\chi^{2}$ test for the categorical variables; $P<0.05$ was considered significant.

\section{Statistical Analysis}

Data were analyzed using stata software version 14. In descriptive statistics, data are presented as mean, standard deviation (SD), frequency, and frequency percentage. In analytical statistics, the Kolmogorov-Smirnov test was performed for the normality of continuous variables and the Schoenfeld residual test was performed to check the proportional hazards $(\mathrm{pH})$ assumption in the simple model and Cox multiple model. To determine difference between the mean of $\mathrm{HB}$ variable data between the two groups (statin vs. non-statin), an independent $t$-test was used for continuous variables such as $\mathrm{PO} 2$ status, body temperature, WBC, platelets, neutrophils and lymphocytes. For non-parametric variables? comparison between groups was performed using Mann-Whitney $U$ test. Fisher's exact test and Chi-square test were used for categorical variables.

Kaplan-Meyer curve was drawn to show the survival time of patients in the two groups--treated with statins and those not treated with statins. Log-Rank test was performed to check the difference in survival time between the two groups of patients. Cox simple regression model was used to determine the factors related to survival time in patients with COVID-19. These analyses were also carried out to test the effect of statin use on the composite outcome. Multivariable Cox regression model was used for variables that were statically significant in the simple Cox regression model. Hazard ratio (HR) and 95\% confidence interval (CI) were reported for each of the variables related to patient survival time in two simple models and Cox multivariable model. The significance level was 0.05 .

\section{RESULTS}

The total number of COVID-19 patients who were included in this study was 583. One hundred sixty-two patients were treated with atorvastatin (statin group) and the remaining 421 were not treated with statin (non-statin group). The mean age of the patients was 60 years and $52.3 \%$ of participants were male. There was no significant relationship between patients' age and statin use. Symptoms of hospitalized patients are listed in Table 1. The most common symptoms included fever, cough, respiratory distress, nausea, and muscle aches. Comorbidities of these patients are listed in Table 2. The most common comorbidity was hypertension (26\%), followed by cardiovascular diseases (including unstable angina, stable angina, myocardial infarction, congestive heart failure and coronary heart disease) (25\%). The prevalence of hypertension was higher in the statin group (33.9 vs. $23.9 \%$ in non-statin group). Cardiovascular diseases were more often in non-statin group ( 27.3 vs. $19.1 \%$ in the statin group). $50 \%$ of patients had $\mathrm{pO} 2<93 \%$, which was similar in both groups. The need for oxygen therapy in the statin group was higher than in the non-statin group (30 vs. $15 \%$ ). The mean time between onset of symptoms and hospitalization of patients was 4.7 days, which was similar in both groups.

Seventy-seven patients $(16.7 \%)$ were admitted to the ICU ward. 28 of these patients were treated with statins while 49 patients were in non-statin group. The rate of admission to ICU did not differ significantly between the two groups $(p=0.8)$, A total of $509(87.5 \%)$ patients were discharged from the hospital, and $73(12.5 \%)$ died. $17(10.4 \%)$ patients who died were in the statin group and $56(13.3 \%)$ were in the non-statin group.

The rate of mechanical ventilation was in all patients $7.5 \%$, but it was higher in the group that was treated with statins (4.9 vs. $3.8 \%$ ). In $49.8 \%$ of patients, CT scan was abnormal and showed serious signs of lung involvement which was higher than in patients treated with statins. WBC count was higher in the group that did not take statins than in the statin group $(p=0.001)$. Statin therapy also reduced neutrophiles count $(p=0.001)$.

The Kaplan-Meyer curve showed that people who were treated with statins had a longer survival time than those who were not treated. Log-rank statistical test indicated that the survival of patients between the two groups (statin and nonstatins) was statistically significant (Figure 1). $14.4 \%(n=44)$ of all deaths were in men. The risk ratio in men was 1.49 higher than women, i.e., survival was lower in men than in women, although the risk ratio in women and men was not significantly different $(95 \% \mathrm{CI}=0.93,2.38)$.

Results of Cox regression were reported as crude and adjusted hazard ratios (HRs) and are presented in Table 2 . They indicated that with 1-year increase in age? the risk of death in COVID-19 patients increased by 1.02 folds $(\mathrm{HR}=1.02, p=0.034)$. Findings in the crude model indicated that the risk of mortality in the statin group was lower than patients who were not treated with statins, in other words, treatment with statin increased survival time $(\mathrm{HR}=0.56,95 \% \mathrm{CI}=(0.32,0.97) p=0.040)$. However, 
TABLE 2 | Data analysis using the crude and adjusted Cox regression models considering mortality as a single outcome.

\begin{tabular}{|c|c|c|c|c|c|c|}
\hline Variable & Level variable & $\begin{array}{c}\text { Death } \\
(n=73,12.5 \%)\end{array}$ & \multicolumn{2}{|c|}{ Crude model } & \multicolumn{2}{|c|}{ Adjusted model } \\
\hline \multirow[t]{2}{*}{ Sex } & Female (n, \%) & 29(10.5) & Baseline & 0.095 & - & - \\
\hline & Male (n, \%) & $44(14.4)$ & $1.49(0.93,2.38)$ & & - & \\
\hline ICU & $\mathrm{ICU}$ & $40(51.9)$ & Baseline & 0.001 & Baseline & 0.117 \\
\hline \multirow[t]{2}{*}{ Atorvastatin } & Yes (n, \%) & $17(10.4)$ & Baseline & 0.040 & Baseline & 0.114 \\
\hline & & & $0.56(0.32,0.97)$ & & $0.51(0.22,1.17)$ & \\
\hline \multicolumn{7}{|l|}{ Sign and symptoms } \\
\hline \multirow[t]{2}{*}{ Fever } & Yes (n, \%) & $25(8.6)$ & Baseline & 0.001 & Baseline & 0.467 \\
\hline & & & $0.44(0.27,0.72)$ & & $1.30(0.64,2.63)$ & \\
\hline Cough & Yes (n, \%) & $22(10.3)$ & Baseline & 0.027 & Baseline & 0.711 \\
\hline \multirow[t]{2}{*}{ Consciousness } & Yes (n, \%) & $10(30.3)$ & Baseline & 0.103 & - & - \\
\hline & & & $1.75(0.89,3.43)$ & & - & \\
\hline \multicolumn{7}{|l|}{ Comorbidities } \\
\hline \multirow[t]{2}{*}{ Diabetes } & Yes (n, \%) & 15 (18.5) & Baseline & 0.133 & - & - \\
\hline & & & $1.54(0.87,2.73)$ & & - & \\
\hline \multirow[t]{2}{*}{ Cardiovascular diseases } & Yes (n, \%) & $26(17.8)$ & Baseline & 0.011 & Baseline & 0y.023 \\
\hline & & & $1.86(1.15,3.01)$ & & $2.37(1.13,4.99)$ & \\
\hline \multirow[t]{2}{*}{ Asthma } & Yes (n, \%) & $12(22.6)$ & Baseline & 0.109 & - & - \\
\hline & & & $1.66(0.89,3.09)$ & & - & \\
\hline Hypertension & Yes (n, \%) & $22(14.1)$ & Baseline & 0.793 & - & - \\
\hline & $>93 \%$ & $16(5.7)$ & $0.41(0.23,0.73)$ & & $0.58(0.22,1.49)$ & \\
\hline Number of breaths & $14-18$ & $4(11.4)$ & Baseline & 0.434 & - & - \\
\hline & $18-22$ & $13(10.6)$ & $0.63(0.20,1.97)$ & & - & \\
\hline & $>22$ & $12(42.8)$ & $1.92(0.60,6.10)$ & 0.266 & - & - \\
\hline CT scan & Yes $(n, \%)$ & $22(13.7)$ & Baseline & 0.874 & - & -- \\
\hline & & & $0.95(0.54,1.68)$ & & - & \\
\hline Opium use & Yes (n, \%) & $8(19.5)$ & Baseline & 0.080 & - & - \\
\hline & & & $1.93(0.92,4.04)$ & & - & \\
\hline SPO2 (mean \pm S.D) & 85.4 & 10.2 & $0.94(0.92,0.96)$ & 0.001 & $0.96(0.92,0.99)$ & 0.035 \\
\hline Body temperature (mean \pm S.D) & 36. & 0.6 & $0.69(0.47,1.01)$ & 0.060 & - & - \\
\hline $\begin{array}{l}\text { The time from the onset of symptoms } \\
\text { to hospitalization (mean } \pm \text { S.D) }\end{array}$ & & & $0.94(0.84,1.04)$ & 0.253 & - & - \\
\hline Laboratory measures & & & & & & \\
\hline WBC (mean \pm S.D) & & & $1.02(1.01,1.04)$ & 0.012 & $1.04(0.93,1.16)$ & 0.459 \\
\hline $\mathrm{HB}($ mean \pm S.D) & 12 & 1.8 & $0.90(0.79,1.03)$ & 0.145 & - & - \\
\hline $\mathrm{PLT}($ mean \pm S.D) & 194 & 87.9 & $0.99(0.99,1.00)$ & 0.399 & - & - \\
\hline Neut (mean \pm S.D) & 78.0 & 14.9 & $1.01(0.99,1.03)$ & 0.191 & - & - \\
\hline Lymph (mean \pm S.D) & 15.3 & 11.8 & $0.97(0.95,1.00)$ & 0.056 & - & - \\
\hline
\end{tabular}


TABLE 2 | Continued

\begin{tabular}{|c|c|c|c|c|c|}
\hline \multirow[t]{2}{*}{ Variable } & \multirow[t]{2}{*}{ Level variable } & \multicolumn{2}{|c|}{ Crude model } & \multicolumn{2}{|c|}{ Adjusted model } \\
\hline & & HR (95\% Cl) & $p$ & HR (95\% Cl) & $p$ \\
\hline RDW (mean \pm S.D) & $15.0 \pm 2.2$ & $1.17(1.06,1.29)$ & 0.001 & $0.91(0.76,1.09)$ & 0.318 \\
\hline MPV (mean \pm S.D) & $10.0 \pm 1.0$ & $0.99(0.79,1.24)$ & 0.941 & - & - \\
\hline BUN (mean \pm S.D) & $23.8 \pm 16.7$ & $1.02(1.01,1.04)$ & 0.001 & $1.01(0.92,1.04)$ & 0.198 \\
\hline Cr (mean \pm S.D) & $1.5 \pm 1.8$ & $1.13(1.01,1.26)$ & 0.025 & $1.18(1.01,1.39)$ & 0.044 \\
\hline CRP (mean \pm S.D) & $1.1 \pm 0.6$ & $1.03(0.75,1.41)$ & 0.835 & - & - \\
\hline
\end{tabular}

$P<0.05$ was considered significant.

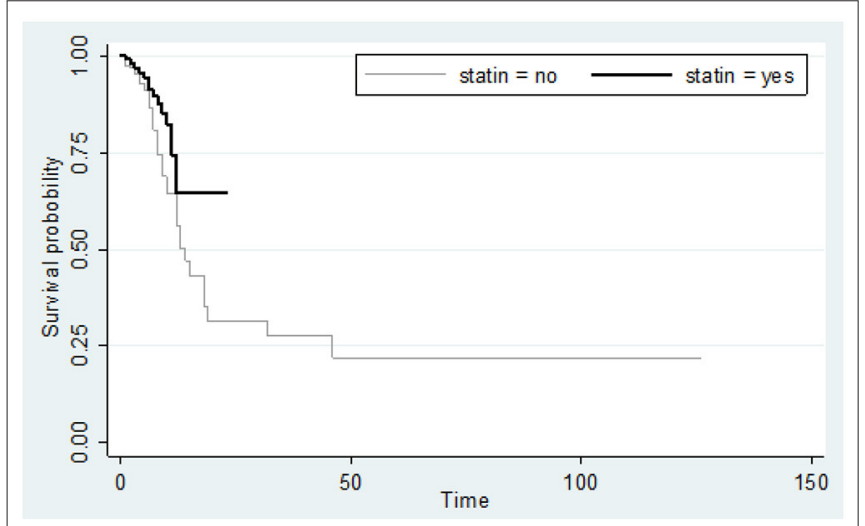

FIGURE 1 | Kaplan-Meier curve comparing the survival time (in days) between statin users and statin non-users considering mortality as an event. Value test log-rank $=4.46 ; p$-value $=0.034$.

the results of Cox multivariable model showed that the reduction of mortality rate in those treated with statin was numerically by $49 \%$ but not statistically significant $[\mathrm{HRs}=0.51,95 \% \mathrm{CI}=$ $(0.22,1.17) p=0.114]$. Mortality rate in patients with COVID-19 who had a history of heart disease was 2.37 times higher than in patients without a history of heart disease $[\mathrm{HR}=2.37,95 \% \mathrm{CI}=$ $(1.13,4.99) p=0.023]$.

Patients who were admitted to ICU and patients who were mechanically ventilated had a higher risk of death than the others, although this difference was not statistically significant.

A secondary analysis was also performed considering a composite outcome comprising intubation, ICU admission, and death. The results of Kaplan-Meyer curve showed that statin use was associated with less composite outcome (Figure 2). Results of Cox regression model are presented in Table 3. The crude model of Cox regression showed patients who used statins were less likely to experience the composite outcome compared with non-statin users [18/162 (11.1\%) vs. 77/421 (18.3\%); HR =0.43, 95\% CI: $0.26,0.73 ; p=0.002)$. The results of the adjusted Cox multivariable model also confirmed the lower occurrence of composite outcome in statin users vs. non-statin users (HR $=0.57,95 \%$ CI: $0.33,0.99 ; p=0.048)$. Age $(\mathrm{HR}=1.02,95 \%$ CI: $1.01,1.03 ; p=0.016)$ and the male gender $(\mathrm{HR}=1.82$,
95\%CI: $1.18,2.83 ; p=0.007)$ were associated with higher rates of composite outcome.

\section{DISCUSSION}

In this retrospective study performed on 583 hospitalized COVID-19 patients, the effects of atorvastatin on mortality, ICU admission, and intubation were investigated. Results of the crude regression model showed that statins reduced mortality. However, this reduction in mortality was not significant in the adjusted model, which might be attributed to the low number of events. When the composite outcome was considered, the results of both crude and adjusted models supported a significant protective effect of statin use. Kaplan-Meyer curve also showed that statin use increased survival of hospitalized patients. A recent study in Shiraz also found that statins reduced mortality in hospitalized COVID-19 patients, although this was not statistically significant (29). In a retrospective study on 13,981 COVID-19 patients in Hubei Province, China, 1,219 patients were treated with statins. In our study, it was found that the survival rate in men was lower than women; in other words, the risk ratio in men was 1.49 times higher than women. This is consistent with the excess mortality observed in men in other populations (30). However, the results of our analyses on the impact of statin therapy were adjusted for potential confounders, including gender.

The present results indicated that statins reduced mortality in these patients. The mortality rate in the statin group was $5.2 \%$, while in the non-statin group, this rate was higher $(9.4 \%)$ (24). In accordance with these data, in a study performed by Masana et al. on 2,157 patients with COVID-19, patients who were treated with statins $(n=581)$ had a lower mortality rate than those who were not treated (31). In contrary to these studies, a cohort study in South Korea on 7,780 COVID19 patients demonstrated that there was no difference in mortality rate between patients treated with statins and those who were not treated (32). In a retrospective cohort study on 2,191 hospitalized COVID-19 patients, statins were also not independently associated with all-cause mortality during follow-up (33).

Another recent retrospective analysis of 4,447 COVID-19 patients admitted to the hospital found that statin use was not associated with altered mortality and found an close to 


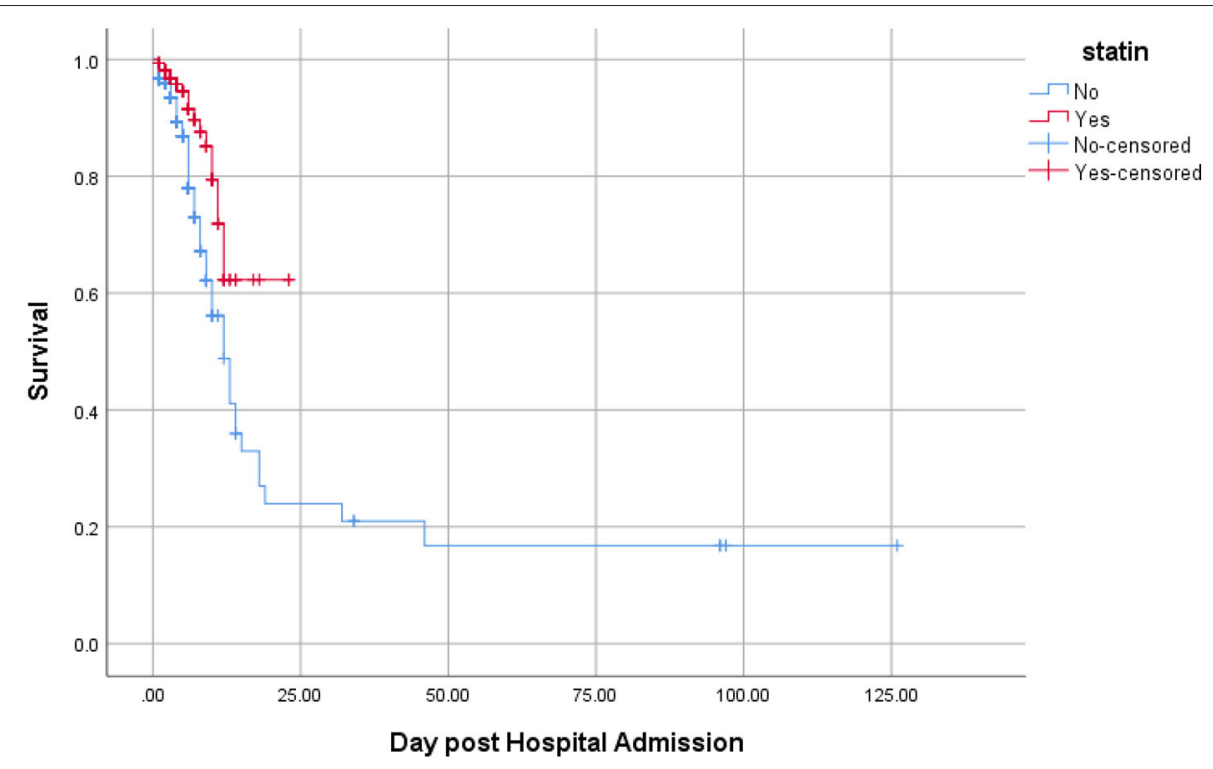

FIGURE 2 | Kaplan-Meier curve comparing the survival time (in days) between statin users and statin nonusers considering the composite of mortality, ICU admission, and intubation as the event. Value test log-rank $=11.06 ; p$-value $=0.001$.

$20 \%$ increased risk of COVID-19 infection (34). It is important to mention the results of some studies in which individuals were treated with statins prior to hospitalization for COVID19 and had therefore lower odds of death, especially those with a history of CVD. For example, a study on 10,541 patients hospitalized with COVID-19 at 104 US hospitals showed that treatment with statins prior to admission was associated with a $>40 \%$ reduction in mortality and a $>25 \%$ reduction in the risk of developing a severe outcome, after controlling for other medication use, comorbid conditions, hospital site and month of admission, and patient demographic characteristics (35). In another retrospective study of 1,014 patients admitted to the hospital because of COVID-19, 454 patients $(44.77 \%)$ were treated with statins prior to hospitalization and such therapy was also associated with significant reduction in mortality (36).

However, the evidence regarding the effects of statins in the treatment of COVID-19 are still controversial. A number of clinical trials and observational studies have reported the protective effects of statins in reducing mortality as well as reducing clinical symptoms in respiratory infections (37). Preclinical studies have reported that statins increase ACE2, an enzyme that facilitates the entry of virus into cells. However, this hypothesis has not been proven in humans. Several studies have suggested that statins improve the outcomes of COVID19 patients by reducing inflammation (38). Anti-inflammatory and immunomodulatory effects of statins are well-known and statins exert their effects by inhibiting the NF-K $\beta$ pathway and by reducing inflammatory mediators such as inflammatory cytokines (IL1, IL6, TNF- $\alpha$ ), CRP, and neutrophils. The results of this study indicated that CRP levels were lower in patients treated with atorvastatin than in those who were not treated with statin. It has been shown that atorvastatin, pravastatin, and rosuvastatin can increase ACE2 levels, which supports the hypothesis that statins inhibit RAS activation, improve vascular remodeling after vascular injury and reduce angiotensin II proinflammatory effects. They can alter CD147 expression, structure, and function by inhibiting its isoprenylation and N-glycosylation, and CD147 is a surface protein that can act as a coronavirus receptor. Statins also suppress NLRP3 inflammasome activation and exactly inflammasome activation in SARS-CoV-2 results in respiratory, $\mathrm{CV}$, gastrointestinal, neurological, renal and ophthalmic manifestations as has been very nicely reviewed in a recently published paper by Torres-Peña et al. (39). However, apart from anti-inflammatory and immunomodulatory effects of statins, statins might deplete cholesterol from cell membranes resulting in coronavirus suppression. Namely, a recently published study showed that SARS-CoV-2 induces cholesterol 25-hydroxylase (25HC) both in vitro and in COVID19 infected patients, via interferon signaling and that $25 \mathrm{HC}$ activation causes a depletion of accessible cholesterol on cell membrane and results in broad anti-coronavirus activity by blocking viral-cell fusion and preventing viral infection of lung epithelial cells (40).

Neutrophil's count, since they are the main cells involved in inflammation, was lower in patients treated with statin than in those who were not treated with a statin. On the other hand, the lymphocyte count in the statin group was higher. This is in accordance with the results of Zhang et al. who reported that statins reduced CRP levels and neutrophils count (24).

Another study on patients with renal failure who had COVID19 found that statins reduced neutrophils count, although this had no effect on mortality (41). One of the aims of the present study was to investigate the effects of statins on the hospitalization of patients with COVID-19 in ICU. Results the aim of this study was also to show whether statins had any 
TABLE 3 | Data analysis using the crude and multiple Cox regression models for the composite outcome comprising mortality, ICU admission and intubation.

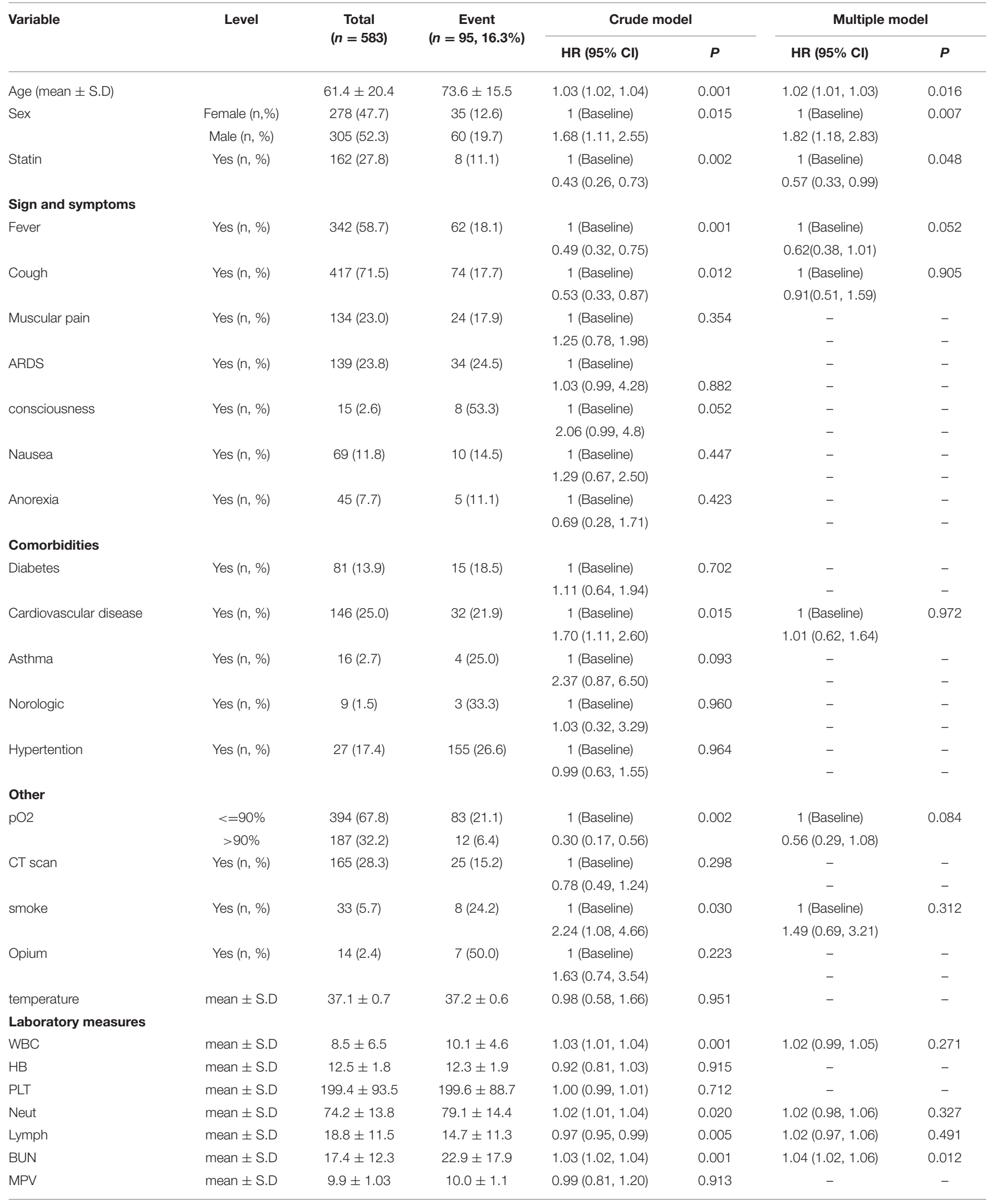


TABLE 3 | Continued

\begin{tabular}{|c|c|c|c|c|c|c|c|}
\hline \multirow[t]{2}{*}{ Variable } & \multirow[t]{2}{*}{ Level } & \multirow{2}{*}{$\begin{array}{c}\text { Total } \\
(n=583)\end{array}$} & \multirow{2}{*}{$\begin{array}{c}\text { Event } \\
(n=95,16.3 \%)\end{array}$} & \multicolumn{2}{|c|}{ Crude model } & \multicolumn{2}{|c|}{ Multiple model } \\
\hline & & & & HR (95\% Cl) & $P$ & HR (95\% Cl) & $\boldsymbol{P}$ \\
\hline $\mathrm{Cr}$ & mean \pm S.D & $1.2 \pm 0.9$ & $1.5 \pm 1.7$ & $1.13(1.02,1.25)$ & 0.016 & $1.18(0.98,1.42)$ & 0.079 \\
\hline CRP & mean \pm S.D & $1.1 \pm 0.7$ & $1.2 \pm 0.7$ & $1.18(0.89,1.54)$ & 0.243 & - & - \\
\hline RDW & mean \pm S.D & $14.1 \pm 1.8$ & $14.9 \pm 2.1$ & $1.14(1.05,1.25)$ & 0.003 & $1.06(0.96,1.18)$ & 0.235 \\
\hline
\end{tabular}

Extended Cox Model; $P<0.05$ was considered significant.

effect on the rate of hospitalization in ICU. The results of this study could not confirm this. A study by Zhang et al. found that statins reduced admission to ICU. A systematic review and metaanalysis also reported that statins reduced admissions to ICU (14). These discrepancies may be related to the type of statistical methods used and the size of the study population. In this study, most of the participants had comorbidities: hypertension and cardiovascular disease, which was consistent with other studies. Prospective studies are needed to determine the possible benefits of statins in the treatment of COVID-19 patients. Several such clinical trials are ongoing.

\section{LIMITATIONS}

Our study as a retrospective study had some limitations. First, because it was retrospective, some medical records of the patients were incomplete. Moreover, the presence of confounding variables caused bias in the results. Therefore, prospective studies should be conducted to obtain more accurate results. Third, this study could only assess the impact of atorvastatin as it was the only statin used by the patients. Hence, it remains unclear if other statins might exert different effects. Finally, the sample size was relatively small to draw a robust conclusion on the impact of statin use on mortality as a single outcome, and larger studies are warranted to explore this further.

\section{CONCLUSION}

Statins with a wide range of pleiotropic effects besides lipid lowering might be useful in the treatment of COVID-19 patients. The anti-inflammatory effects of statins can contribute to the prevention of cytokine storm and lung damage. The results of this study also suggested the protective effects of statins in reducing

\section{REFERENCES}

1. Dreher M, Kersten A, Bickenbach J, Balfanz P, Hartmann B, Cornelissen C, et al. The characteristics of 50 hospitalized COVID-19 patients with and without ARDS. Deutsches Ärzteblatt Int. (2020) 117:271. doi: 10.3238/arztebl.2020.0271

2. Hariyanto TI, Kurniawan A. Statin therapy did not improve the inhospital outcome of coronavirus disease 2019 (COVID-19) infection. Diabetes Metabolic Syndr. (2020) 14:1613-5. doi: 10.1016/j.dsx.2020.08.023 inflammation. Statins seem to reduce the composite of mortality, ICU admission, and intubation in COVID-19 patients, although prospective studies are needed in larger populations to confirm this effect.

\section{DATA AVAILABILITY STATEMENT}

The raw data supporting the conclusions of this article will be made available by the authors, upon a reasonable request without undue reservation.

\section{ETHICS STATEMENT}

The studies involving human participants were reviewed and approved by the Ethics Committee of Birjand University of Medical Sciences (IR.BUMS.REC.1400.073). The patients/participants provided their written informed consent to participate in this study.

\section{AUTHOR CONTRIBUTIONS}

HKo, MB, and AS: conceptualization. HKo, HKh, ZK, AN, HM, and HF: data collection and writing-original draft. MA, ŽR, TK, $\mathrm{MB}$, and AS: writing-review and editing. All authors approved the manuscript.

\section{FUNDING}

This work was supported by Russian Science Foundation (Grant \# 22-25-00498).

\section{ACKNOWLEDGMENTS}

The authors are thankful to the Birjand University of Medical Sciences, Birjand, Iran for its support.
3. Ferretti G, Bacchetti T, Sahebkar A. Effect of statin therapy on paraoxonase-1 status: A systematic review and meta-analysis of 25 clinical trials. Prog Lipid Res. (2015) 60:50-73. doi: 10.1016/j.plipres.2015.08.003

4. Gorabi AM, Kiaie N, Pirro M, Bianconi V, Jamialahmadi T, Sahebkar A. Effects of statins on the biological features of mesenchymal stem cells and therapeutic implications. Heart Fail Rev. (2021) 26:1259-72. doi: 10.1007/s10741-020-09929-9

5. Parizadeh SMR, Azarpazhooh MR, Moohebati M, Nematy M, Ghayour-Mobarhan M, Tavallaie S, et al. Simvastatin therapy reduces 
prooxidant-antioxidant balance: Results of a placebo-controlled cross-over trial. Lipids. (2011) 46:333-40. doi: 10.1007/s11745-010-3517-x

6. Sahebkar A, Serban C, Mikhailidis DP, Undas A, Lip GYH, Muntner P, et al. Association between statin use and plasma d-dimer levels: A systematic review and meta-analysis of randomised controlled trials. Thrombosis Haemostasis. (2015) 114:546-57. doi: 10.1160/TH14-11-0937

7. Shakour N, Ruscica M, Hadizadeh F, Cirtori C, Banach M, Jamialahmadi T, et al. Statins and C-reactive protein: In silico evidence on direct interaction. Arch Med Sci. (2020) 16:1432-9. doi: 10.5114/aoms.2020.100304

8. Sohrevardi SM, Nasab FS, Mirjalili MR, Bagherniya M, Tafti AD, Jarrahzadeh $\mathrm{MH}$, et al. Effect of atorvastatin on delirium status of patients in the intensive care unit: A randomized controlled trial. Arch Med Sci. (2021) 17:1423. doi: 10.5114/aoms.2019.89330

9. Reiner Ž, Hatamipour M, Banach M, Pirro M, Al-Rasadi K, Jamialahmadi T, et al. Statins and the COVID-19 main protease: In silico evidence on direct interaction. Arch Med Sci: AMS. (2020) 16:490. doi: 10.5114/aoms.2020.94655

10. Castiglione V, Chiriacò M, Emdin M, Taddei S, Vergaro G. Statin therapy in COVID-19 infection. Eur Heart J Cardiovasc Pharmacother. (2020) 6:258-9. doi: 10.1093/ehjcvp/pvaa042

11. Bahrami A, Parsamanesh N, Atkin SL, Banach M, Sahebkar A. Effect of statins on toll-like receptors: a new insight to pleiotropic effects. Pharmacol Res. (2018) 135:230-8. doi: 10.1016/j.phrs.2018.08.014

12. Khalifeh M, Penson PE, Banach M, Sahebkar A. Statins as anti-pyroptotic agents. Arch Med Sci. (2021) 17:1414-7. doi: 10.5114/aoms/141155

13. Franco-Peláez JA, Esteban-Lucia L, Chacón MdláZ, Pello-Lázaro AM, Rodriguez AMV, Roca LN, et al. Statin use is associated with reduced mortality after respiratory viral infection. ERJ Open Res. (2021) 7:365. doi: $10.1183 / 23120541.00365-2020$

14. Vahedian-Azimi A, Mohammadi SM, Beni FH, Banach M, Guest PC, Jamialahmadi T, et al. Improved COVID-19 ICU admission and mortality outcomes following treatment with statins: A systematic review and meta-analysis. Arch Med Sci. (2021) 17:579-95. doi: 10.5114/aoms/ 132950

15. Ganjali S, Bianconi V, Penson PE, Pirro M, Banach M, Watts GF, et al. Statins, COVID-19, and coronary artery disease: killing two birds with one stone. Metabolism. (2020) 113:154375. doi: 10.1016/j.metabol.2020. 154375

16. Radenkovic D, Chawla S, Pirro M, Sahebkar A, Banach M. Cholesterol in relation to covid-19: Should we care about it? J Clin Med. (2020) 9:1-9. doi: $10.3390 / \mathrm{jcm} 9061909$

17. Vahedian-Azimi A, Mohammadi SM, Banach M, Beni FH, Guest PC, AlRasadi K, et al. Improved COVID-19 outcomes following statin therapy: an updated systematic review and meta-analysis. BioMed Res Int. (2021) 2021:1772. doi: $10.1155 / 2021 / 1901772$

18. Vahedian-Azimi A, Rahimibashar F, Najafi A, Kidde J, Shahriary A, Shojaei S, et al. Associastion of in-hospital use of statins, aspirin, and renin-angiotensin-aldosterone inhibitors with mortality and ICU admission due to COVID-19. Adv Experi Medici Biol. (2021) 2021:205-14. doi: 10.1007/978-3-030-71697-4_17

19. Torres-Peña JD, Pérez-Belmonte LM, Fuentes-Jiménez F, Carmona MDL, Pérez-Martinez P, López-Miranda J, et al. Prior treatment with statins is associated with improved outcomes of patients with COVID19: data from the SEMI-COVID-19 Registry. Drugs. (2021) 81:685-95. doi: $10.1007 / \mathrm{s} 40265-021-01498-\mathrm{x}$

20. Ulrich H, Pillat MM. CD147 as a target for COVID-19 treatment: suggested effects of azithromycin and stem cell engagement. Stem Cell Rev Rep. (2020) 16:434-40. doi: 10.1007/s12015-020-09976-7

21. Gareeva D, Musin T, Pavlov V, Davtyan P, Ishmetov VS, Plotnikova M, et al. Statins: unexpected help in COVID-19. Arterial Hyperten. (2020) 26:509-17. doi: 10.18705/1607-419X-2020-26-5-509-517

22. Middeldorp S, Coppens $M$, van Haaps TF, Foppen M, Vlaar AP, Müller MC, et al. Incidence of venous thromboembolism in hospitalized patients with COVID-19. J Thrombosis Haemostasis. (2020) 18:1995-2002. doi: $10.1111 /$ jth. 14888

23. Gong J, Dong H, Xia QS, Huang ZY, Wang DK, Zhao Y, et al. Correlation analysis between disease severity and inflammation-related parameters in patients with COVID-19: a retrospective study. BMC Infect Dis. (2020) 20:963. doi: 10.1186/s12879-020-05681-5
24. Zhang X-J, Qin J-J, Cheng X, Shen L, Zhao Y-C, Yuan Y, et al. Inhospital use of statins is associated with a reduced risk of mortality among individuals with COVID-19. Cell Metabo. (2020) 32:176-87.e4. doi: 10.1016/j.cmet.2020.06.015

25. Lee KCH, Sewa DW, Phua GC. Potential role of statins in COVID-19. Int $J$ Infect Dis. (2020) 96:615-7. doi: 10.1016/j.ijid.2020.05.115

26. Michaud V, Deodhar M, Arwood M, Al Rihani SB, Dow P, Turgeon J. ACE2 as a therapeutic target for COVID-19; its role in infectious processes and regulation by modulators of the RAAS system. J Clin Med. (2020) 9:2096. doi: $10.3390 / \mathrm{jcm} 9072096$

27. Fajgenbaum DC, Rader DJ. Teaching old drugs new tricks: statins for COVID19? Cell Metabolism. (2020) 32:145-7. doi: 10.1016/j.cmet.2020.07.006

28. Momtazi-Borojeni AA, Banach M, Reiner Ž, Pirro M, Bianconi V, Al-Rasadi $\mathrm{K}$, et al. Interaction between coronavirus S-protein and human ACE2: Hints for exploring efficient therapeutic targets to treat COVID-19. Angiology. (2021) 72:122-30. doi: 10.1177/0003319720952284

29. Peymani P, Dehesh T, Aligolighasemabadi F, Sadeghdoust M, Kotfis K, Ahmadi M, et al. Statins in patients with COVID-19: a retrospective cohort study in Iranian COVID-19 patients. Translational Med Commun. (2021) 6:1-14. doi: 10.1186/s41231-021-00082-5

30. Nielsen J, Nørgaard SK, Lanzieri G, Vestergaard LS, Moelbak K. Sex-differences in COVID-19 associated excess mortality is not exceptional for the COVID-19 pandemic. Sci Rep. (2021) 11:20815. doi: 10.1038/s41598-021-00213-w

31. Masana L, Correig E, Rodríguez-Borjabad C, Anoro E, Arroyo JA, Jericó C, et al. Effect of statin therapy on SARS-CoV-2 infection-related mortality in hospitalized patients. Eur Heart J Cardiovasc Pharmacother. (2020) 2:pvaa128. doi: $10.1093 /$ ehjcvp/pvaa128

32. Oh TK, Song I, Jeon Y-T. Statin therapy and the risk of COVID-19: a cohort study of the national health insurance service in South Korea. J Personal Med. (2021) 11:116. doi: 10.3390/jpm11020116

33. Rey JR, JL ML, ÁM IM, Rodríguez SOR, Castrejón-Castrejón S, ArbasRedondo E, et al. Influence of statin treatment in a cohort of patients admitted for COVID-19. Med Clin. (2021). doi: 10.1016/j.medcli.2021. 07.003. [Epub ahead of print].

34. Ayeh SK, Abbey EJ, Khalifa BA, Nudotor RD, Osei AD, Chidambaram V, et al. Statins use and COVID-19 outcomes in hospitalized patients. PLoS ONE. (2021) 16:e0256899. doi: 10.1371/journal.pone.0256899

35. Daniels LA-O, Ren J, Kumar K, Bui QM, Zhang J, Zhang X, et al. Relation of prior statin and anti-hypertensive use to severity of disease among patients hospitalized with COVID-19: Findings from the American Heart Association's COVID-19 Cardiovascular Disease Registry. PLoS ONE. (2021) 16:e0254635. doi: 10.1371/journal.pone.0254635

36. Lohia P, Kapur S, Benjaram S, Mir T. Association between antecedent statin use and severe disease outcomes in COVID-19: A retrospective study with propensity score matching. J Clin Lipidol. (2021) 15:451-9. doi: 10.1016/j.jacl.2021.03.002

37. Gupta A, Madhavan MV, Poterucha TJ, DeFilippis EM, Hennessey JA, Redfors B, et al. Association between antecedent statin use and decreased mortality in hospitalized patients with COVID-19. Nat Commun. (2021) 12:1-9. doi: 10.1038/s41467-021-21553-1

38. Vuorio AJ, Kovanen PT. Statins as adjuvant therapy for COVID-19 to calm the stormy immunothrombosis and beyond. Front Pharmacol. (2020) 11:2265. doi: 10.3389/fphar.2020.579548

39. Torres-Peña JD, Katsiki N, Perez-Martinez P. Could Statin therapy be useful in patients with coronavirus disease 2019 (COVID-19)? Front Cardiovasc Med. (2021) 8:775749. doi: $10.3389 /$ fcvm.2021.7 75749

40. Wang S, Li W, Hui H, Tiwari SK, Zhang Q, Croker BA, et al. Cholesterol 25-Hydroxylase inhibits SARS-CoV-2 and other coronaviruses by depleting membrane cholesterol. EMBO J. (2020) 9:e106057. doi: $10.15252 / \mathrm{embj} .2020106057$

41. Bifulco M, Gazzerro P. Statin therapy in COVID-19 infection: much more than a single pathway. Eur Heart J Cardiovasc Pharmacother. (2020) 6:410-1. doi: 10.1093/ehjcvp/pvaa055

Conflict of Interest: MB speakers bureau: Abbott/Mylan, Abbott Vascular, Actavis, Akcea, Amgen, Biofarm, KRKA, MSD, Polpharma, Sanofi-Aventis, 
Servier and Valeant; consultant to Abbott Vascular, Akcea, Amgen, Daichii Sankyo, Esperion, Freia Pharmaceuticals, Lilly, MSD, Polfarmex, Resverlogix, Sanofi-Aventis; Grants from Sanofi and Valeant. ŽR has received honoraria from Sanofi-Aventis and Novartis.

The remaining authors declare that the research was conducted in the absence of any commercial or financial relationships that could be construed as a potential conflict of interest.

Publisher's Note: All claims expressed in this article are solely those of the authors and do not necessarily represent those of their affiliated organizations, or those of the publisher, the editors and the reviewers. Any product that may be evaluated in this article, or claim that may be made by its manufacturer, is not guaranteed or endorsed by the publisher.

Copyright (c) 2022 Kouhpeikar, Khosaravizade Tabasi, Khazir, Naghipour, Mohammadi Moghadam, Forouzanfar, Abbasifard, Kirichenko, Reiner, Banach and Sahebkar. This is an open-access article distributed under the terms of the Creative Commons Attribution License (CC BY). The use, distribution or reproduction in other forums is permitted, provided the original author(s) and the copyright owner(s) are credited and that the original publication in this journal is cited, in accordance with accepted academic practice. No use, distribution or reproduction is permitted which does not comply with these terms. 